\title{
STAR PRODUCT AND STAR EXPONENTIAL
}

\author{
TOSHIO TOMIHISA AND AKIRA YOSHIOKA
}

Communicated by Ivaïlo M. Mladenov

\begin{abstract}
Here we extend the star products by means of complex symmetric matrices. In this way we obtain a family of star products. Next we consider the star exponentials with respect to these star products, and finally we obtain several interesting identities.
\end{abstract}

\section{Introduction}

In order to express the elements in Weyl algebra, we need to fix the ordering of the generators in monomials because of their non-commutativity. The ordering yields a linear isomorphism between the Weyl algebra and the space of all complex polynomials and the isomorphism naturally induces an associative product in the space of polynomials. This product is called a star product. For example, the normal ordering induces normal product, anti-normal ordering induces the anti-normal product and the Weyl ordering yields the Moyal product, respectively.

The so obtained star product algebra is isomorphic to the Weyl algebra, and then these are mutually isomorphic (see for example Omori-Maeda-MiyazakiYoshioka [1]). As an extension of these star products, Omori-Maeda-MiyazakiYoshioka [2] introduced a family of star products parameterized by the space of all complex symmetric matrices. Then a geometric picture is given for the family parameterized by the space of complex matrices. The family forms an algebraic bundle over the space of all complex symmetric matrices.

When one has to exponentiate elements in the star product algebra, one needs to deal with the infinite sum of the power series with respect to the Plank constant. Then, in order to discuss the convergence of these series it is necessary to introduce a topology and to take the completion of the star product algebra. A typical topology is a formal power series topology. Under this topology, the star product is well-defined as a formal power series in Plank constant with coefficients of smooth functions. Hence one can exponentiate any element of the star product algebra. Also, under this topology, it is proved that star products can be considered 
on any symplectic manifold (see [3] and [4]), and further on any Poisson manifold (cf. [5]).

On the other hand, in [6], a different topology is proposed. This permits to deal with the Plank constant as a number, not as a formal parameter. Families of seminorms and their topologies are introduced into the space of complex polynomials. Taking the completion, we obtain a family of Fréchet spaces consisting of holomorphic functions. It is shown that the star products are well-defined for certain classes of these topological spaces and each of them becomes a Fréchet topological algebra. In these spaces, star exponentials are investigated for the elements of the algebra, especially for the linear elements and quadratic elements, by means of geometric methods.

In this paper, a survey of these topological spaces and some main results concerning the star exponentials together with some concrete examples is presented. The paper is organized as follows. First we explain the general setting by introducing the concept of $q$-number functions. Then we consider the examples of star exponential and its application.

This paper is based mainly on the talk given by the second author at Conference on Geometry, Integrability and Quantization in Varna 2009.

\section{A Family of Star Products}

In this section, we introduce a family of star products parameterized by the space of all complex symmetric matrices. Using the intertwiners, we give a geometric picture for the family of star products.

\subsection{Star Products}

For simplicity, we consider star products of two variables $\left(u_{1}, u_{2}\right)$. The general case for $2 m$ variables $\left(u_{1}, u_{2}, \cdots, u_{2 m}\right)$ is similar.

Let us fix the skew symmetric matrix

$$
J=\left(\begin{array}{cc}
0 & 1 \\
-1 & 0
\end{array}\right)
$$

For an arbitrary complex symmetric matrix $K \in \mathcal{S}_{\mathbb{C}}(2)$ we put

$$
\Lambda=J+K=\left(\begin{array}{ll}
\lambda_{11} & \lambda_{12} \\
\lambda_{21} & \lambda_{22}
\end{array}\right)
$$


and consider a bi-derivation acting on the pair $p_{1}\left(u_{1}, u_{2}\right), p_{2}\left(u_{1}, u_{2}\right)$ in the space $\mathcal{P}\left(\mathbb{C}^{2}\right)$ of the complex polynomials by the formula

$$
p_{1}(\overleftarrow{\partial} \Lambda \vec{\partial}) p_{2}=p_{1}\left(\sum_{k, l=1}^{2} \lambda_{k l} \overleftarrow{\partial}_{u_{k}} \vec{\partial}_{u_{l}}\right) p_{2}=\sum_{k, l=1}^{2} \lambda_{k l} \partial_{u_{k}} p_{1} \partial_{u_{l}} p_{2}
$$

Now we define a star product $*_{K}$ on the space of complex polynomials $p_{1}\left(u_{1}, u_{2}\right)$, $p_{2}\left(u_{1}, u_{2}\right)$ by

$$
\begin{aligned}
p_{1} *_{K} p_{2}= & p_{1} \exp \left(\frac{\mathrm{i} \hbar}{2} \overleftarrow{\partial} \Lambda \vec{\partial}\right) p_{2}=p_{1} p_{2}+\frac{\mathrm{i} \hbar}{2} p_{1}(\overleftarrow{\partial} \Lambda \vec{\partial}) p_{2} \\
& +\cdots+\frac{1}{n !}\left(\frac{\mathrm{i} \hbar}{2}\right)^{n} p_{1}(\overleftarrow{\partial} \Lambda \vec{\partial})^{n} p_{2}+\cdots
\end{aligned}
$$

Proposition 1. For an arbitrary complex symmetric matrix $K \in \mathcal{S}_{\mathbb{C}}(2)$ the star product $*_{K}$ is associative on the space of all complex polynomials $\mathcal{P}\left(\mathbb{C}^{2}\right)$.

We remark here that the definition of star products $*_{K}$ is an extension of star products given by standard ordering problems. For example, if we put $K=0$, then the product becomes the Moyal product. Similarly, for $K=\left(\begin{array}{ll}0 & 1 \\ 1 & 0\end{array}\right)$ we obtain the normal product and for $K=\left(\begin{array}{rr}0 & -1 \\ -1 & 0\end{array}\right)$ the anti-normal product.

These are products on polynomials and the so obtained algebras are all isomorphic to the Weyl algebra. For an arbitrary $K \in \mathcal{S}_{\mathbb{C}}(2)$, the product $*_{K}$ satisfies the canonical commutation relations

$$
\left[u_{k}, u_{l}\right]_{*_{K}}=u_{k} *_{K} u_{l}-u_{l} *_{K} u_{k}=\mathrm{i} \hbar \delta_{k l}, \quad k, l=1,2
$$

and hence it follows that all algebras $\left(\mathcal{P}\left(\mathbb{C}^{2}\right), *_{K}\right)$ are isomorphic to the Weyl algebra $W_{2}$ with two generators $u_{1}, u_{2}$. Actually, we have algebra isomorphisms $I_{K_{1}}^{K_{2}}$ between any two of these algebras $\left(\mathcal{P}\left(\mathbb{C}^{2}\right), *_{K_{1}}\right),\left(\mathcal{P}\left(\mathbb{C}^{2}\right), *_{K_{2}}\right)$. The algebra isomorphism (intertwiners)

$$
I_{K_{1}}^{K_{2}}:\left(\mathcal{P}\left(\mathbb{C}^{2}\right), *_{K_{1}}\right) \rightarrow\left(\mathcal{P}\left(\mathbb{C}^{2}\right), *_{K_{2}}\right)
$$

are explicitly given by

$$
I_{K_{1}}^{K_{2}}(p)=\exp \left(\frac{\mathrm{i} \hbar}{4}\left(K_{2}-K_{1}\right) \partial^{2}\right) p
$$


where

$$
\left(K_{2}-K_{1}\right) \partial^{2}=\sum_{k l=1}^{2}\left(K_{2}-K_{1}\right)_{k l} \partial_{u_{k}} \partial_{u_{l}} .
$$

We have the relations

Proposition 2. i) $I_{K_{2}}^{K_{3}} I_{K_{1}}^{K_{2}}=I_{K_{1}}^{K_{3}}$, ii) $\left(I_{K_{1}}^{K_{2}}\right)^{-1}=I_{K_{2}}^{K_{1}}$.

By differentiating the intertwiner with respect to $K$, we obtain an infinitesimal intertwiner at $K$

$$
\nabla_{\kappa}(p)=\frac{\mathrm{d}}{\mathrm{d} t} I_{K}^{K+t \kappa}(p)_{\left.\right|_{t=0}}=\frac{\mathrm{i} \hbar}{4} \kappa \partial^{2} p
$$

where

$$
\kappa \partial^{2} p=\sum_{i, j}^{2} \kappa_{i j} \partial_{i} \partial_{j} p .
$$

Then the infinitesimal intertwiner satisfies

$$
\nabla_{\kappa}\left(p_{1} *_{K} p_{2}\right)=\nabla_{\kappa}\left(p_{1}\right) *_{K} p_{2}+p_{1} *_{K} \nabla_{\kappa}\left(p_{2}\right)
$$

for any $p_{1}\left(u_{1}, u_{2}\right), p_{2}\left(u_{1}, u_{2}\right) \in \mathcal{P}\left(\mathbb{C}^{2}\right)$.

\section{3. $q$-number Polynomials}

In the star product algebras $\left\{\left(\mathcal{P}\left(\mathbb{C}^{2}\right), *_{K}\right)\right\}_{K \in \mathcal{S}_{\mathbb{C}}(2)}$, the algebras $\left(\mathcal{P}\left(\mathbb{C}^{2}\right), *_{K_{1}}\right)$ and $\left(\mathcal{P}\left(\mathbb{C}^{2}\right), *_{K_{2}}\right)$ are mutually isomorphic by the intertwiner $I_{K_{1}}^{K_{2}}$ and the elements $p_{1} \in\left(\mathcal{P}\left(\mathbb{C}^{2}\right), *_{K_{1}}\right)$ and $p_{2} \in\left(\mathcal{P}\left(\mathbb{C}^{2}\right), *_{K_{2}}\right)$ are identified when

$$
p_{2}=I_{K_{1}}^{K_{2}}\left(p_{1}\right) \text {. }
$$

A naturally geometric picture follows for the family of star product algebras $\left\{\left(\mathcal{P}\left(\mathbb{C}^{2}\right), *_{K}\right)\right\}_{K \in \mathcal{S}_{\mathbb{C}}(2)}$. To describe it, we introduce an algebra bundle over $\mathcal{S}_{\mathbb{C}}(2)$ whose fibres consist of the Weyl algebra in the following way.

1. Algebra bundle. We consider the trivial bundle

$$
\pi: \mathbb{P}=\mathcal{P}\left(\mathbb{C}^{2}\right) \times \mathcal{S}_{\mathbb{C}}(2) \rightarrow \mathcal{S}_{\mathbb{C}}(2)
$$

whose fibre over $K \in \mathcal{S}_{\mathbb{C}}(2)$ consists of the star product algebra

$$
\pi^{-1}(K)=\left(\mathcal{P}\left(\mathbb{C}^{2}\right), *_{K}\right)
$$


2. Flat connection and parallel translation. On this bundle, we regard the infinitesimal intertwiner $\nabla$ as a flat connection and the intertwiner $I_{K_{1}}^{K_{2}}$ as its parallel translation.

We consider a section $\tilde{p} \in \Gamma(\mathbb{P})$ of this bundle satisfying

$$
\tilde{p}\left(K_{2}\right)=I_{K_{1}}^{K_{2}}\left(\tilde{p}\left(K_{1}\right)\right)
$$

This means that $\tilde{p}$ is parallel

$$
\nabla_{\kappa} \tilde{p}(K)=0
$$

\section{3. q-number polynomial}

We denote by $\mathcal{P}(\mathbb{P})$ the space of all parallel sections, and call an element $\tilde{p} \in \mathcal{P}(\mathbb{P}) q$-number polynomial.

Due to the identities $I_{K_{2}}^{K_{3}} I_{K_{1}}^{K_{2}}=I_{K_{1}}^{K_{3}}$ and $\left(I_{K_{1}}^{K_{2}}\right)^{-1}=I_{K_{2}}^{K_{1}}$ the intertwiners naturally induce the star product $*$ on $\mathcal{P}(\mathbb{P})$. Then the algebra $(\mathcal{P}(\mathbb{P}), *)$ is regarded as a geometric realization of the Weyl algebra.

\section{4. $q$-number Functions}

Here we introduce a locally convex topology into the star product algebras by means of a system of semi-norms.

Then we take the completion of the algebras and consider the star exponentials.

\subsection{Topology}

We introduce a topology into $\mathcal{P}\left(\mathbb{C}^{2}\right)$ by a system of semi-norms in the following way. Let $\rho$ be a positive number. For every $s>0$ we define a semi-norm in the space of polynomials by

$$
|p|_{s}=\sup _{u \in \mathbb{C}^{2}}\left|p\left(u_{1}, u_{2}\right)\right| \exp \left(-s|u|^{\rho}\right)
$$

Then the system of semi-norms $\left\{|\cdot|_{s}\right\}_{s>0}$ defines a locally convex topology $\mathcal{T}_{\rho}$ on $\mathcal{P}\left(\mathbb{C}^{2}\right)$. 


\subsection{Fréchet Space $\mathcal{E}_{\rho}\left(\mathbb{C}^{2}\right)$}

Definition 3. We take the completion of $\mathcal{P}\left(\mathbb{C}^{2}\right)$ with respect to the topology $\mathcal{T}_{\rho}$, we obtain a Fréchet space $\mathcal{E}_{\rho}\left(\mathbb{C}^{2}\right)$.

Proposition 4. For a positive number $\rho$, the Fréchet space $\mathcal{E}_{\rho}$ consists of entire functions on the complex plane $\mathbb{C}^{2}$ with finite semi-norm for every $s>0$, namely

$$
\mathcal{E}_{\rho}\left(\mathbb{C}^{2}\right)=\left\{f \in \mathcal{H}\left(\mathbb{C}^{2}\right) ;|f|_{s}<+\infty, \text { for all } s>0\right\} .
$$

Continuity for the case $0<\rho \leq 2$.

As to the continuity of star products and intertwiners, the Fréchet space $\mathcal{E}_{\rho}\left(\mathbb{C}^{2}\right)$, $0<\rho \leq 2$ is very good, namely, we have the following

Theorem 5. On $\mathcal{E}_{\rho}\left(\mathbb{C}^{2}\right), 0<\rho \leq 2$ every product $*_{K}$ is continuous, and every intertwiner $I_{K_{1}}^{K_{2}}:\left(\mathcal{E}_{\rho}\left(\mathbb{C}^{2}\right), *_{K_{1}}\right) \rightarrow\left(\mathcal{E}_{\rho}\left(\mathbb{C}^{2}\right), *_{K_{2}}\right)$ is continuous.

Continuity as a bimodule for the case $\rho>2$.

As to the spaces $\mathcal{E}_{\rho}\left(\mathbb{C}^{2}\right)$ for $\rho>2$, the situation is no so good, but still we have the following.

Theorem 6. If $\rho>2$, take $\rho^{\prime}>0$ such that

$$
\frac{1}{\rho^{\prime}}+\frac{1}{\rho}=1
$$

then every star product $*_{K}$ defines a continuous bilinear product

$$
*_{K}: \mathcal{E}_{\rho}\left(\mathbb{C}^{2}\right) \times \mathcal{E}_{\rho^{\prime}}\left(\mathbb{C}^{2}\right) \rightarrow \mathcal{E}_{\rho}\left(\mathbb{C}^{2}\right), \mathcal{E}_{\rho^{\prime}}\left(\mathbb{C}^{2}\right) \times \mathcal{E}_{\rho}\left(\mathbb{C}^{2}\right) \rightarrow \mathcal{E}_{\rho}\left(\mathbb{C}^{2}\right)
$$

This means that $\left(\mathcal{E}_{\rho}\left(\mathbb{C}^{2}\right), *_{K}\right)$ is a continuous $\mathcal{E}_{\rho^{\prime}}\left(\mathbb{C}^{2}\right)$-bimodule.

\section{3. $q$-number Functions}

The case $0<\rho \leq 2$.

Due to the previous theorem, we can introduce a similar concept as $q$-number polynomials into the Fréchet spaces. Namely, the star product $*_{K}$ is well defined on $\mathcal{E}_{\rho}\left(\mathbb{C}^{2}\right)$ and then we can consider the trivial bundle

$$
\pi: \mathbb{E}_{\rho}=\mathcal{E}_{\rho}\left(\mathbb{C}^{2}\right) \times \mathcal{S}_{\mathbb{C}}(2) \rightarrow \mathcal{S}_{\mathbb{C}}(2)
$$


with fibre over the point $K \in \mathcal{S}_{\mathbb{C}}(2)$ consisting of

$$
\pi^{-1}(K)=\left(\mathcal{E}_{\rho}\left(\mathbb{C}^{2}\right), *_{K}\right) .
$$

The intertwiners $I_{K_{1}}^{K_{2}}$ are well defined for any $K_{1}, K_{2} \in \mathcal{S}_{\mathbb{C}}(2)$ and then the bundle $\mathbb{E}_{\rho}$ has a flat connection $\nabla$ and the parallel translation is the intertwiner.

The space of flat sections of the bundle denoted by $\mathcal{F}_{\rho}$ is naturally equipped with the star product $*$ and can be regarded as a completion of the Weyl algebra $W_{2}$.

Remark to the case $\rho>2$.

For the case $\rho>2$, it is not clear at present whether the intertwiners are welldefined and whether the product $*_{K}$ are well defined. However the flat connection $\nabla$ is still well defined on $\pi: \mathbb{E}_{\rho}=\mathcal{E}_{\rho}\left(\mathbb{C}^{2}\right) \times \mathcal{S}_{\mathbb{C}}(2) \rightarrow \mathcal{S}_{\mathbb{C}}(2)$, so we can define a space $\mathcal{F}_{\rho}$ of all parallel sections of this bundle even for $\rho>2$.

For $\rho>2$, we are trying to extend the product ${ }^{*} K_{K}$ and also the intertwiners $I_{K_{1}}^{K_{2}}$ by means of some regularizations, for example, Borel-Laplace transform, or finite part regularization. We hope to construct such a concept in the near future.

\section{Star Exponential}

The space of all $q$-number functions $\mathcal{F}_{\rho}$ is a complete topological algebra for all $\rho, 0<\rho \leq 2$ (even for $\rho>2$ under some regularization). We can consider exponential element

$$
\exp _{*} t\left(\frac{H}{\mathrm{i} \hbar}\right)=\sum_{n=0}^{\infty} \frac{t^{n}}{n !} \underbrace{\frac{H}{\mathrm{i} \hbar} * \cdots * \frac{H}{\mathrm{i} \hbar}}_{n}
$$

in this algebra.

For a $q$-number polynomial $H \in \mathcal{P}(\mathbb{P})$, we define the star exponential $\exp _{*} t(H / \mathrm{i} \hbar)$ by the differential equation

$$
\frac{\mathrm{d}}{\mathrm{d} t} \exp _{*} t\left(\frac{H}{\mathrm{i} \hbar}\right)=\frac{H}{i \hbar} * \exp _{*} t\left(\frac{H}{\mathrm{i} \hbar}\right),\left.\exp _{*} t\left(\frac{H}{\mathrm{i} \hbar}\right)\right|_{t=0}=1 .
$$

Remark 7. We set the Fréchet space

$$
\mathcal{E}_{\rho+}\left(\mathbb{C}^{2}\right)=\cap_{\lambda>\rho} \mathcal{E}_{\lambda}\left(\mathbb{C}^{2}\right)
$$

and we denote by $\mathfrak{E}_{\rho+}$ the corresponding bundle and by $\mathcal{F}_{\rho+}$ the space of the flat sections of this bundle. 
When $H \in \mathcal{P}(\mathbb{P})$ is a linear element, then $\exp _{*} t\left(\frac{H}{\mathrm{i} \hbar}\right)$ belongs to the good space $\mathcal{F}_{1+}\left(\subset \mathcal{F}_{2}\right)$.

On the other hand, the most interesting case is provided by the quadratic form $H \in \mathcal{P}(\mathbb{P})$. In this case we can solve the differential equation explicitly, but the star exponential belongs to the space $\mathcal{F}_{2+}$, which is difficult to treat at present.

Although general theory related to the space $\mathcal{F}_{2+}$ is not yet established, we present a concrete example of the star exponential of the quadratic forms and its application.

\section{Examples}

Let us vary the parameter $K \in \mathcal{S}_{\mathbb{C}}(2)$ as for some $K$ we can obtain interesting identities in the algebra of ${ }_{K}$ product.

The linear case

Here we consider a liner $q$-number polynomial. It can be written in a general form as

$$
H=a_{1} u+a_{2} v=\langle\boldsymbol{a}, \boldsymbol{u}\rangle, \quad a_{1}, a_{2} \in \mathbb{C} .
$$

Star exponential in $H$ belongs to the space of $q$-number functions $\mathcal{F}_{1+}$. The star exponential $\exp _{*} t\left(\frac{H}{\mathrm{i} \hbar}\right)$ at $K$, which is denoted by $\exp _{*_{K}} t\left(\frac{H}{\mathrm{i} \hbar}\right)$, is explicitly given by the formula

$$
\exp _{*_{K}} t\left(\frac{H}{\mathrm{i} \hbar}\right)=\exp \frac{1}{4 \hbar}\langle\boldsymbol{a} K, \boldsymbol{a}\rangle \exp \frac{1}{\mathrm{i} \hbar}\langle\boldsymbol{a}, \boldsymbol{u}\rangle .
$$

Hence, if the real part satisfies an inequality like

$$
\operatorname{Re} \frac{1}{4 \hbar}\langle\boldsymbol{a} K, \boldsymbol{a}\rangle<0
$$

and the term $\exp \frac{t^{2}}{4 \hbar}\langle\boldsymbol{a} K, \boldsymbol{a}\rangle$ is rapidly decreasing with respect to $t$, we can consider the integral

$$
\int_{-\infty}^{\infty} \mathrm{e}^{-\mathrm{e}^{t}} \exp _{*_{K}} t\left(z+\frac{H}{\mathrm{i} \hbar}\right) \mathrm{d} t .
$$

Then we can define the star gamma function by

$$
\Gamma_{*}(z)=\int_{-\infty}^{\infty} \mathrm{e}^{-\mathrm{e}^{t}} \exp _{*} t\left(z+\frac{H}{\mathrm{i} \hbar}\right) \mathrm{d} t .
$$

This is evaluated at every $K$ and the value $\Gamma_{*_{K}}(z)$ of the star gamma function at $K$ is given by the integral, where $K$ satisfies the condition (22). 
We have the identity for $K$ satisfying (22)

$$
\Gamma_{*_{K}}(z+1)=\left(z+\frac{H}{\mathrm{i} \hbar}\right) *_{K} \Gamma_{*_{K}}(z) .
$$

\section{Quadratic case}

In this paragraph, we construct a Clifford algebra by means of the star exponential $\exp _{*} t\left(\frac{2 u * v}{\mathrm{i} \hbar}\right)$ for certain $K$. In what follows, we describe a rough sketch of the construction.

First we consider a generic point in $\mathcal{S}_{\mathbb{C}}(2)$, i.e.,

$$
K=\left(\begin{array}{cc}
\tau^{\prime} & \kappa \\
\kappa & \tau
\end{array}\right) \in \mathcal{S}_{\mathbb{C}}(2)
$$

Next in the star product $*_{K}$ algebra, we write the generator $u=u_{1}, v=u_{2}$ satisfying

$$
[u, v]_{*_{K}}=-\mathrm{i} \hbar .
$$

Then the star exponential of $H=2 u * v$ is explicitly given at a general point $K$ as

$$
\begin{aligned}
\exp _{*_{K}} t & \left(\frac{2 u * v}{\mathrm{i} \hbar}\right) \\
& =\frac{2 \mathrm{e}^{-t}}{\sqrt{D}} \exp \left[\frac{\mathrm{e}^{t}-\mathrm{e}^{-t}}{\mathrm{i} \hbar D}\left(\left(\mathrm{e}^{t}-\mathrm{e}^{-t}\right) \tau u^{2}+2 \Delta u v+\left(\mathrm{e}^{t}-\mathrm{e}^{-t}\right) \tau^{\prime} v^{2}\right)\right]
\end{aligned}
$$

where

$$
D=\triangle^{2}-\left(\mathrm{e}^{t}-\mathrm{e}^{-t}\right) \tau^{\prime} \tau, \quad \triangle=\mathrm{e}^{t}+\mathrm{e}^{-t}-\kappa\left(\mathrm{e}^{t}-\mathrm{e}^{-t}\right)
$$

In the sequel, we assume $\tau^{\prime}=0$, that is, we take the point

$$
K=\left(\begin{array}{cc}
0 & \kappa \\
\kappa & \tau
\end{array}\right)
$$

We have a limit

$$
\lim _{t \rightarrow-\infty} \varpi_{00}=\exp _{*_{K}} t\left(\frac{2 u * v}{\mathrm{i} \hbar}\right)=\frac{2}{1+\kappa} \exp \left(-\frac{1}{\mathrm{i} \hbar(1+\kappa)}\left(2 u v-\frac{\tau}{1+\kappa} u^{2}\right)\right)
$$

which we call a vacuum.

Then we state 
Lemma 8. i) $\varpi_{00} *_{K} \varpi_{00}=\varpi_{00}$, ii) $v *_{K} \varpi_{00}=\varpi_{00} *_{K} u=0$.

Putting $t=\pi \mathrm{i}$, we have the identity

$$
\exp _{*_{K}} \pi \mathrm{i}\left(\frac{2 u * v}{\mathrm{i} \hbar}\right)=1
$$

Using

$$
v *_{K}\left(u *_{K} v\right)=\left(v *_{K} u\right) *_{K} v=\left(u *_{K} v+\mathrm{i} \hbar\right) *_{K} u
$$

we see that the star exponential satisfies

$$
\begin{array}{rl}
v *_{K} \exp _{*_{K}} & t\left(\frac{2 u * v}{\mathrm{i} \hbar}\right)=\exp _{*_{K}} t\left(\frac{2 v * u}{\mathrm{i} \hbar}\right) *_{K} v \\
= & \exp _{*_{K}} t\left(\frac{2 u * v+2 \mathrm{i} \hbar}{\mathrm{i} \hbar}\right) *_{K} v=\mathrm{e}^{2 t} \exp _{*_{K}} t\left(\frac{2 u * v}{\mathrm{i} \hbar}\right) *_{K} v .
\end{array}
$$

Then the integral $\frac{1}{2} \int_{-\infty}^{0} \exp _{*_{K}} t\left(\frac{2 v * u}{\mathrm{i} \hbar}\right) \mathrm{d} t$ converges and we can define

$$
\frac{1}{2} \int_{-\infty}^{0} \exp _{*_{K}} t\left(\frac{2 v * u}{\mathrm{i} \hbar}\right) \mathrm{d} t=\left(v *_{K} u\right)_{+}^{-1}
$$

and

$$
\stackrel{\circ}{v}=u *_{K}\left(v *_{K} u\right)_{+}^{-1} .
$$

Then we have

Lemma 9. The element $\stackrel{\circ}{v}$ is the right inverse of $v$ satisfying

$$
v *_{K} \stackrel{\circ}{v}=1, \quad \stackrel{\circ}{v} *_{K} v=1-\varpi_{00} .
$$

Now we fix an integer $l$. By putting

$$
t=t_{l}=\frac{\pi \mathrm{i}}{2^{l}}
$$

we obtain $2^{l}$ roots of the unity

$$
\Omega_{l}=\exp _{*_{K}} \frac{\pi \mathrm{i}}{2^{l}}\left(\frac{2 u * v}{\mathrm{i} \hbar}\right), \quad \varpi_{l}=\exp 2\left(\frac{\pi \mathrm{i}}{2^{l}}\right)
$$

such that

$$
\Omega_{l *_{K}}^{2^{l}}=\underbrace{\Omega_{l} *_{K} \cdots *_{K} \Omega_{l}}_{2^{l}}=1, \quad \varpi_{l}^{2^{l}}=1 .
$$

Finally we have 
Lemma 10. We have the following relation

$$
\Omega_{l *_{K}}^{k} *_{K} u_{*_{K}}^{m} *_{K} \varpi_{00} *_{K} v_{*_{K}}^{m}=\varpi_{l}^{k m} u_{*_{K}}^{m} *_{K} \varpi_{00} *_{K} v_{*_{K}}^{m} .
$$

Now we take appropriate complex numbers $a_{0}, a_{1}, \cdots, a_{2^{l}-1}$ so that the element

$$
E=\sum_{k=0}^{2^{l}-1} a_{k} \Omega_{l * K}^{k}
$$

obeys to the identities

$$
\begin{aligned}
E *_{K} u_{*_{K}}^{m} *_{K} \varpi_{00} *_{K} & v_{*_{K}}^{m} \\
& = \begin{cases}*_{K} u_{*_{K}}^{m} *_{K} \varpi_{00} *_{K} v_{*_{K}}^{m} & \cdots 0 \leq m \leq 2^{l-1}-1 \\
0 & \cdots 2^{l-1} \leq m \leq 2^{l}-1 .\end{cases}
\end{aligned}
$$

We see that this is equivalent to

$$
\sum_{k=0}^{2^{l}-1} a_{k} \varpi_{l}^{k m}=\left\{\begin{array}{c}
1 \cdots 0 \leq m \leq 2^{l-1}-1 \\
0 \cdots 2^{l-1} \leq m \leq 2^{l}-1
\end{array}\right.
$$

The complex numbers $a_{0}, a_{1}, \cdots, a_{2^{l}-1}$ are uniquely determined by these equations. Then we have

Lemma 11. The element E satisfies

$$
E *_{K} E=1
$$

and the element $F=1-E$ satisfies

$$
F *_{K} F=1, \quad E *_{K} F=F *_{K} E=0 .
$$

Further we have

Lemma 12. $E *_{K}(v)_{*_{K}}^{2^{l-1}}=(v)_{*_{K}}^{2^{l-1}} *_{K} F, \quad(\stackrel{\circ}{v})_{*_{K}}^{2^{l-1}} *_{K} F=E *(\stackrel{\circ}{v})_{*_{K}}^{2^{l-1}}$ where

$$
(v)_{*_{K}}^{2^{l-1}}=\underbrace{v *_{K} \cdots *_{K} v}_{2^{l-1}} \quad \text { and }(\stackrel{\circ}{v})_{*_{K}}^{2^{l-1}}=\underbrace{\stackrel{\circ}{v} *_{K} \cdots *_{K}}_{2^{l-1}} \stackrel{\circ}{v} .
$$


Finally we can set

$$
\xi=E *_{K}(v)_{*_{K}}^{2^{l-1}}, \quad \eta=(\stackrel{\circ}{v})_{*_{K}}^{2^{l-1}} *_{K} F .
$$

Then we have

Theorem 13. The elements $\xi$ and $\eta$ of the $*_{K}$ product algebra satisfies the identities

$$
\begin{aligned}
& \xi *_{K} \xi=\eta *_{K} \eta=0 \\
& \xi *_{K} \eta+\xi *_{K} \eta=1 .
\end{aligned}
$$

\section{Acknowledgements}

The authors would like to express their thanks to Professor Ivaïlo M. Mladenov and Professor Gaetano Vilasi for the useful discussions and encouragement. The second author was partially supported by the Grant-in-Aid for Scientific Research (\# 21540096).

\section{References}

[1] Omori H., Maeda Y., Miyazaki N. and Yoshioka A., Strange Phenomena Related to Ordering Problems in Quantization, J. Lie Theory 13 (2003) 481510.

[2] Omori H., Maeda Y., Miyazaki N. and Yoshioka A., Star Exponential Functions as Two-valued Elements, In: The Breadth of Symplectic and Poisson Geometry, Progr. Math. 232, J. Marsden and T. Ratiu (Eds), Birkhauser, Boston, 2005, pp.483-492.

[3] Omori H., Maeda Y. and Yoshioka A., Weyl Manifolds and Deformation Quantization, Adv. Math. 85 (1991) 224-255.

[4] Fodosov B., A Simple Geometrical Construction of Deformation Quantization, J. Diff. Geom. 40 (1994) 213-238.

[5] Kontsevich M., Deformation Quantization of Poisson Manifolds, Lett. Math. Phys. 66 (2003) 157-216.

[6] Omori H., Maeda Y., Miyazaki N. and Yoshioka A., Deformation Quantization of Fréchet Poisson Algebras, - Convergence of the Moyal Product - , In: Conference Moshé Flato 1999, Quantizations, Deformations and Symmetries vol III, Math. Phys. Studies 22, Kluwer, 2000 pp 233-246. 
Toshio Tomihisa

Department of Mathematics

Faculty of Science

Tokyo University of Science

162-8601, Kagurazaka 1-3, Shinjyuku-ku

Tokyo, JAPAN

E-mail address: j1107705@ed.kagu.tus.ac.jp

Akira Yoshioka

Department of Mathematics

Faculty of Science

Tokyo University of Science

162-8601, Kagurazaka 1-3, Shinjyuku-ku

Tokyo, JAPAN

E-mail address: yoshioka@rs.kagu.tus.ac.jp 